\title{
ESTRATEGIA PARA FORTALECER LAS CAPACIDADES DE INVESTIGACIÓN EN SALUD EN UNIVERSIDADES PÚBLICAS REGIONALES: ROL DEL CANON Y DEL INSTITUTO NACIONAL DE SALUD
}

\author{
Franco Romaní1,a, César Cabezas ${ }^{2, b}$, Manuel Espinoza ${ }^{3, b}$, Gabriela Minaya ${ }^{1, b}$, José Huaripata ${ }^{1, c}$ \\ Juan Manuel Ureta ${ }^{1, \mathrm{~d}}$, Myriam Yazuda ${ }^{3, \mathrm{e}}$, María del Carmen Gastañaga ${ }^{3, \mathrm{f}}$, María Luz Miraval ${ }^{3, \mathrm{e}}$, \\ Juan Pablo Aparco ${ }^{4, g}$, Elizabeth Anaya ${ }^{1, h}$, José Castro,a, Silvia Esquivel ${ }^{6, c}$
}

\section{RESUMEN}

\begin{abstract}
El desarrollo de investigación científica en salud requiere de un sistema de investigación sostenido, articulado y coherente con las prioridades de investigación; este sistema de investigación implica la existencia de financiamiento tanto interno como externo, y de disponer recursos humanos competentes. El canon minero es un derecho constitucional, parte de él ha sido destinado para el desarrollo de investigación científica y aplicada por las universidades públicas (UP). El Instituto Nacional de Salud (INS) tiene entre sus funciones promover, gestionar y difundir el desarrollo de investigación en salud a nivel nacional. Como parte de estas funciones se conformó un equipo técnico para brindar asesoría técnica a las UP para el desarrollo de investigación con fondos del canon minero, mediante la adecuación local de protocolos de investigación promovidos por el INS y asumidos por los docentes-investigadores de la universidad. El presente artículo tiene como objetivo describir la realidad de investigación de las UP peruanas que cuentan con fondos del canon minero, y presentar la intervención que el INS viene realizando para fortalecer las capacidades de investigación, iniciando con desarrollar propuestas de investigación que potencialmente puedan obtener financiamiento de parte del canon.
\end{abstract}

Palabras clave: Fuentes de financiación de investigación; Universidades; Minería; Perú (fuente: DeCS BIREME).

\section{STRATEGY TO STRENGTHEN HEALTH RESEARCH CAPABILITIES IN REGIONAL PUBLIC UNIVERSITIES: THE ROLE OF THE MINING CANON AND THE INSTITUTO NACIONAL DE SALUD}

\begin{abstract}
The development of scientific health research requires a sustained and articulated research system that is consistent with the research priorities, as well as both internal and external funding, and availability of competent human resources. The Mining Canon, a constitutional right, has been partly used to foster applied scientific research in public universities (PU). In addition, the National Health Institute (INSTITUTO NACIONAL DE SALUD - INS) is devoted, among others, to promoting, managing and disseminating health research development at a national level. As part of these activities, a technical team was created to provide technical assistance to PU for research development using Mining Canon funds by making local adjustments to research protocols promoted by the INS and assumed by the professors-researchers at the Universities. This article aims at describing the reality of research at Peruvian public universities that have access to Mining Canon funds, as well as to elaborate on the work the INS is carrying out in order to strengthen research capabilities, starting with the development of research proposals that could potentially be funded by the Mining Canon.
\end{abstract}

Key words: Support of research; Universities; Mining; Peru (source: MeSH NLM).

\footnotetext{
Oficina General de Investigación y Transferencia Tecnológica, Instituto Nacional de Salud. Lima, Perú.

Instituto Nacional de Salud. Lima, Perú.

Centro Nacional de Salud Pública, Instituto Nacional de Salud. Lima, Perú.

Centro Nacional de Nutrición y Alimentación, Instituto Nacional de Salud. Lima, Perú.

Oficina General de Asesoría Técnica, Instituto Nacional de Salud. Lima, Perú.

Agencia Adventista para el Desarrollo de Recursos (ADRA). Lima, Perú.

Médico cirujano; ${ }^{\mathrm{b}}$ médico infectólogo; ${ }^{\mathrm{c}}$ enfermero; ${ }^{\mathrm{d}}$ médico epidemiólogo; ${ }^{\mathrm{e}}$ médico patólogo clínico; ${ }^{\mathrm{f}}$ médico magister en gestión y auditorías ambientales; $\mathrm{g}$ licenciado en Nutrición; ${ }^{\mathrm{h}}$ biólogo

Recibido: 09-08-12 Aprobado: 17-10-12
}

Citar como: Romaní F, Cabezas C, Espinoza M, Minaya G, Huaripata J, Ureta JM, et al. Estrategia para fortalecer las capacidades de investigación en salud en universidades públicas regionales: rol del canon y del Instituto Nacional de Salud. Rev Peru Med Exp Salud Publica. 2012;29(4):549-54. 


\section{PANORAMA GENERAL DEL CANON}

El canon es un porcentaje del total de ingresos y rentas que obtiene el Estado por la explotación económica que realizan las empresas privadas de los recursos naturales no renovables, es además un derecho amparado en la constitución (1); su objetivo es brindar a los gobiernos regionales y locales recursos económicos para promover el desarrollo de inversiones que mejoren la calidad de vida de su población ${ }^{(2-4)}$. La distribución del canon está determinada por el Ministerio de Economía y Finanzas, según el cual el $25 \%$ le corresponde a los gobiernos regionales de donde se explota el recurso natural; el $40 \%$, a las municipalidades departamentales; el $25 \%$, a las municipalidades provinciales y el $10 \%$ le corresponde al distrito productor donde se encuentre el recurso extraído ${ }^{(5)}$. Actualmente, la Ley del Canon señala que $20 \%$ de los recursos asignados a los gobiernos regionales por concepto de canon será entregado a las universidades públicas (UP) de su circunscripción, para su uso en investigación científica y tecnológica que potencien el desarrollo regional ${ }^{(6,7)}$.

Existen seis tipos de canon: minero, petrolero, gasífero, hidroenergético, pesquero y forestal (2,3); cada uno de ellos con un porcentaje diferente de aportación sobre sus impuestos a la renta; el canon minero corresponde el 50\% del total de ingresos y rentas obtenidos por el Estado por el aprovechamiento de los recursos minerales metálicos y no metálicos por parte de la empresa privada ${ }^{(3)}$. Otra fuente de recursos, producto de la explotación minera son las "regalías mineras"; según la Ley 29788, estas son la contraprestación económica que las empresas mineras pagan al Estado por la explotación de recursos minerales metálicos y no metálicos, es calculada sobre la utilidad operativa trimestral de los sujetos de la actividad minera ${ }^{(8)}$, el $5 \%$ del total recaudado bajo este concepto es destinado para las UP de la región donde se explota el recurso natural.
Los recursos recibidos por las UP por concepto de canon, sobre-canon y regalía minera pueden utilizarlo en financiar y cofinanciar investigación científica y tecnológica (9); es decir, investigaciones de ciencia aplicada relacionadas con salud pública y prevención de enfermedades endémicas; sanidad agropecuaria; preservación de la biodiversidad, y utilización de energías renovables y procesos productivos; además, con dicho fondo se puede financiar proyectos de inversión públicos vinculados directamente con los fines de la universidad y para el desarrollo de su infraestructura y equipamiento hasta un límite máximo del $50 \%$. No se permite el uso de estos recursos para el pago de remuneraciones o retribuciones de cualquier tipo al personal docente ${ }^{(10)}$. Además, desde el año 2012 se puede destinar hasta un $20 \%$ de los recursos para actividades relacionadas con procesos de acreditación universitaria ${ }^{(11)}$.

\section{CANON MINERO Y DESEMPEÑO DE LAS UNIVERSIDADES EN LA PRODUCCIÓN CIENTÍFICA}

Para el año 2011, las regiones con mayor cantidad de recursos por canon minero fueron Ancash, Arequipa y La Libertad, quienes recibieron en conjunto 1887 millones de soles (mds) ${ }^{(12)}$. Por tanto, las UP de dichas regiones reciben un presupuesto considerable; las universidades más beneficiadas se detallan en la Tabla 1 .

Un análisis realizado en 2008, encontró que las UP regionales solo habían ejecutado el $26,7 \%$ del total recibido por concepto de canon en los últimos cinco años ${ }^{(13)}$. Si bien la transferencia del canon minero a las universidades busca el desarrollo de investigación científica y tecnológica a favor del desarrollo regional, su implementación parece no haber considerado la escasez

Tabla 1. Transferencia de canon minero a universidades de las doce regiones con mayor ingreso por canon, año 2011.

\begin{tabular}{|c|c|c|c|c|c|c|}
\hline Región & $\begin{array}{c}\text { Canon } \\
\text { minero } \\
\text { (millones S/.) }\end{array}$ & Universidad nacional & $\begin{array}{l}\text { Facultad de } \\
\text { Medicina }\end{array}$ & $\begin{array}{c}\text { Facultad de } \\
\text { ciencias de } \\
\text { la salud* }\end{array}$ & $\begin{array}{c}\text { Facultad } \\
\text { de ciencias } \\
\text { biológicas }\end{array}$ & $\begin{array}{c}\text { Monto del canon para } \\
\text { universidad } \\
\text { (millones de } \mathrm{S} / . \text {.) }\end{array}$ \\
\hline Ancash & 756,0 & UN Santiago Antúnez de Mayolo & No & Sí & No & 151,2 \\
\hline Arequipa & 662,6 & UN San Agustín & Sí & Sí & Sí & 132,5 \\
\hline La Libertad & 459,3 & UN Trujillo & Sí & Sí & Sí & 91,9 \\
\hline Cajamarca & 417,6 & UN de Cajamarca & Sí & Sí & No & 83,5 \\
\hline Moquegua & 392,5 & UN de Moquegua & No & No & No & 78,5 \\
\hline Tacna & 350,1 & UN de Jorge Basadre Grohmann & Sí & Sí & Sí & 70,0 \\
\hline Puno & 307,1 & UN del Altiplano & Sí & Sí & Sí & 61,4 \\
\hline Ica & 201,9 & UN San Luis Gonzaga de Ica & Sí & Sí & Sí & 40,3 \\
\hline Pasco & 181,7 & UN Daniel Alcides Carrión & No & Sí & No & 36,3 \\
\hline Cusco & 170,0 & UN San Antonio Abad & Sí & Sí & Sí & 34,0 \\
\hline Ayacucho & 57,4 & UN San Cristóbal de Huamanga & No & Sí & Sí & 11,5 \\
\hline Huancavelica & 8,5 & UN de Huancavelica & No & Sí & No & 1,7 \\
\hline Total & 3965,2 & & & & & 793,0 \\
\hline
\end{tabular}

*Comprende Facultad de Obstetricia y Enfermería. 
de competencias en investigación en los profesionales de las universidades, ni la poca idoneidad del sistema organizacional universitario para la investigación.

Sobre esto último, un indicador importante es la producción científica de las universidades. En el periodo 20002008, Perú ha mostrado un incremento de publicaciones científicas de 179 en el año 2000 a 453 en el año 2008; sin embargo, nuestra producción para el año 2008 siguió siendo menor que la de otros países de Latinoamérica, como Brasil (con 26482 publicaciones), México (8262) y Argentina (6197) ${ }^{(14)}$. A pesar de este incremento, la producción científica de las UP que reciben canon entre el 2006-2010 ha sido baja; la UN San Antonio Abad del Cusco es la de mayor producción con 94 publicaciones en revistas científicas indizadas en la base de datos SCOPUS; en tanto que las UN de Cajamarca, Huancavelica, Ayacucho, Ancash y Pasco han publicado menos de diez artículos ${ }^{(18)}$

Otro aspecto a tomar en cuenta es la colaboración internacional $(\mathrm{Cl})$, expresada como el porcentaje de publicaciones elaboradas con instituciones de otros países; las UN de Ancash y Pasco desarrollaron el $100 \%$ de sus publicaciones con Cl; la UN San Antonio Abad y de Trujillo tuvieron $\mathrm{Cl}$ en más del $90 \%$ de sus publicaciones. Este detalle pone de manifiesto que sin colaboración, ya sea internacional o de otras instituciones nacionales con mayor experticia en investigación, las UP regionales tendrían incluso una menor producción científica que la actual.

Otro aspecto importante es la calidad de las publicaciones; el indicador CCP (calidad científica promedio o impacto normalizado) permite comparar la calidad de la investigación procedente de instituciones de diferente tamaño y con perfiles de investigación distintos, se calcula a partir del número medio de citas de las publicaciones de la institución con respecto al número medio de citas de la producción mundial en un mismo periodo y área temàtica, el $1 Q$ es otro indicador que mide el porcentaje de publicaciones en revistas del primer cuartil del Journal Citation Report (JCR), en la Tabla 2 se detallan la producción de las UP peruanas.

Un análisis concluye que el canon minero no se ha invertido adecuadamente en investigación debido a las débiles e inconstantes políticas universitarias en investigación; las pobres competencias para la gestión institucional de la investigación; la fuerte influencia de organización burocrática en la investigación, y la escasez a nivel regional de investigadores universitarios competentes, con adecuada formación científico-ética y con experiencia ${ }^{(12)}$; todas estas debilidades se afianzan en un precario sistema nacional de investigación y en la pobre articulación de los institutos especializados de investigación con las universidades a nivel regional.
Tabla 2. Ranking y producción científica de universidades públicas peruanas, periodo 2006-2010.

\begin{tabular}{|c|c|c|c|c|c|}
\hline $\begin{array}{l}\text { Ranking en } \\
\text { Latinoamérica }\end{array}$ & Universidad & $\mathrm{PC}$ & $\mathrm{Cl}$ & $\mathrm{CCP}$ & $1 Q$ \\
\hline 138 & $\begin{array}{l}\text { UN Mayor } \\
\text { de San Marcos* }\end{array}$ & 502 & 60,2 & 0,8 & 39,0 \\
\hline 348 & UN San Antonio Abad & 94 & 97,9 & 1,5 & 61,7 \\
\hline 422 & UN San Agustín & 59 & 78,0 & 0,7 & 33,9 \\
\hline 480 & UN de Trujillo & 45 & 91,1 & 1,0 & 46,7 \\
\hline 623 & UN San Luis Gonzaga & 20 & 50,0 & 0,7 & 35,0 \\
\hline 655 & UN del Altiplano & 17 & 76,5 & 1,4 & 64,7 \\
\hline 556 & $\begin{array}{l}\text { UN Jorge } \\
\text { Basadre Grohmann }\end{array}$ & 17 & 29,4 & 0,3 & 17,7 \\
\hline 811 & UN de Cajamarca & 8 & 50,0 & 0,4 & 25,0 \\
\hline 870 & UN de Huancavelica & 6 & 83,3 & 0,1 & 0 \\
\hline 872 & $\begin{array}{l}\text { UN San Cristóbal de } \\
\text { Huamanga }\end{array}$ & 6 & 83,3 & 0,8 & 50,0 \\
\hline 1023 & $\begin{array}{l}\text { UN Daniel } \\
\text { Alcides Carrión }\end{array}$ & 3 & 100 & 1,9 & 66,7 \\
\hline 1028 & $\begin{array}{l}\text { UN Santiago } \\
\text { Antúnez de Mayolo }\end{array}$ & 3 & 100 & 0,6 & 33,3 \\
\hline
\end{tabular}

PC: producción científica

$\mathrm{Cl}$ : colaboración internacional

CCP: calidad científica promedio

1Q: porcentaje de publicaciones en revistas del primer cuartil del Journal Citation Report

*Universidad pública que no recibe canon, se muestran como referencia

Fuente: Ranking Iberoamericano SIR 2012.

\section{ESTRATEGIA PARA EL DESARROLLO DE PROTOCOLOS DE INVESTIGACIÓN EN SALUD}

Frente a este problema, el Instituto Nacional de Salud (INS) ha desarrollado un plan de intervención denominado "Fortalecimiento de las universidades públicas con canon para la implementación de las prioridades nacionales y regionales de investigación en salud", plan que considera una serie de estrategias relacionadas directamente con la formulación de protocolos de investigación; entre ellas, el fortalecimiento de la participación de las UP en la construcción del Sistema Nacional de Investigación en Salud y en la Red Nacional de Bioética (RENABIP); la asistencia técnica a las UP en el fortalecimiento de la gestión de la investigación en salud; la asistencia técnica para la organización de fondos concursables del canon minero para investigación en salud, y el fomento de la formulación de proyectos de investigación para implementación de las prioridades de investigación en salud.

Una estrategia para hacer efectivo el plan en el corto plazo fue la conformación de un equipo de trabajo multidisciplinario para la asistencia técnica a las UP en el desarrollo de protocolos de investigación con miras a obtener financiamiento del canon minero ${ }^{(19)}$, dicho equipo elaboró un plan de trabajo específico entre el INS y las 
universidades con el objetivo de promover la realización de proyectos de investigación en salud multidisciplinarios e inter institucionales, apoyar a la consolidación de la masa crítica de investigadores en salud y colaborar con el sector salud en la identificación y búsqueda de soluciones a los principales problemas de salud del país.

\section{ETAPAS Y FASES DE LA ESTRATEGIA}

La estrategia constó de dos etapas, una primera de abogacía, en la cual se identificaron a UP beneficiarias de recursos del canon minero y se logró contactar con funcionarios y autoridades con capacidad de decisión en actividades de investigación de cada una de ellas. Se emprendió el proceso de sensibilización para involucrar la participación y compromiso de sus autoridades para el planeamiento, desarrollo y ejecución de investigación en salud. Para facilitar el desarrollo de protocolos, el INS se comprometió a promover protocolos de investigación seleccionados y con aprobación por comités de investigación y de ética; para ello se seleccionaron 57 proyectos que podrían realizarse como investigación multicéntrica y multidisciplinaria; de estos protocolos finalmente 15 fueron seleccionados debido a que respondían a las prioridades nacionales y regionales de investigación. Posteriormente, producto de las actividades de abogacía, se definieron que las primeras universidades en ser asistidas serían las de la regiones Cajamarca, Tacna y Cusco. Un análisis adicional que tomó en consideración las particularidades de cada ámbito geográfico determinó la selección de ocho protocolos de investigación.

La segunda etapa de Asistencia técnica para el desarrollo de protocolos de investigación presenta las siguientes fases:

Feria de proyectos de investigación. Desarrollada mediante exposiciones y presentación de posters de las investigaciones seleccionadas, las cuales ya contaban con aprobación por comités científicos y de ética; esta actividad estuvo a cargo de los investigadores, luego se conformaron equipos integrados por docentes e investigadores de la UP los cuales asumieron alguno de los proyectos promocionados por el INS, los que se detallan en la Tabla 3.

Presentación de objetivos de la asistencia técnica y metodología. Fase durante la cual se expusieron las bases establecidas por la UP para el proceso de fondo concursable; la cartera de programas y servicios del INS para el desarrollo de la investigación en salud, especialmente de la Red Nacional de Bioética, la Red Nacional de Investigación en Salud y del Plan de Fortalecimiento de la Investigación en Universidades; además de la presentación de la metodología de trabajo de la asistencia técnica para la elaboración de protocolos de investigación.

Formación de los equipos de investigación. Se solicitó a los docentes e investigadores que formaran equipos de investigación según su experticia y competencias profesionales y seleccionaran preferentemente alguno de los proyectos presentados. En caso existiera algún equipo ya conformado con algún protocolo avanzado, se verificó que el problema de investigación estuviera dentro de las prioridades de investigación regional.

Asesoría técnica para la formulación de protocolos de investigación. En esta fase se realizaron talleres de trabajo con los grupos de investigación conformados por los docentes e investigadores de la UP y un asesor del INS. Existieron dos situaciones en esta fase: Situación 1, en la cual se adopta un protocolo propuesto por el INS; en dicho caso, se realizó la adaptación del protocolo a la realidad de la región según el formato de presentación establecido en las bases del fondo concursable; y Situación 2, en la cual se formuló un protocolo de investigación desarrollado por los docentes e investigadores de la UP, en este caso se realizó el desarrollo del protocolo desde el inicio según

Tabla 3. Protocolos de investigación presentados en la feria de proyectos en las universidades públicas intervenidas.

\begin{tabular}{|c|c|c|c|c|}
\hline N. ${ }^{\circ}$ & Protocolo de investigación & Cajamarca & Tacna & Cusco \\
\hline 1 & Dinámica de transmisión de peste en cuatro regiones endémicas del Perú 2010-2014. & $\mathrm{X}$ & $\mathrm{X}$ & $\mathrm{X}$ \\
\hline 2 & Enfermedad de Carrión en gestantes de tres zonas endémicas del Perú. & $\mathrm{X}$ & $\mathrm{X}$ & $\mathrm{X}$ \\
\hline 3 & $\begin{array}{l}\text { Evaluación del impacto del Programa Integral de Nutrición (PIN)- Subprograma escolar po- } \\
\text { blación preescolar. }\end{array}$ & $\mathrm{X}$ & $\mathrm{X}$ & $\mathrm{X}$ \\
\hline 4 & $\begin{array}{l}\text { Evaluación del impacto del Programa Integral de Nutrición (PIN)-Diseño Procesos y efectos } \\
\text { en el estado nutricional de la gestante y su recién nacido. }\end{array}$ & $\mathrm{X}$ & $\mathrm{X}$ & $\mathrm{X}$ \\
\hline 6 & $\begin{array}{l}\text { Prevalencia de factores de riesgo de enfermedades no transmisibles en estudiantes de cole- } \\
\text { gios, universidades y mujeres de comedores populares en Lima, Callao, La Libertad y Arequipa. }\end{array}$ & $X$ & $X$ & $X$ \\
\hline 7 & $\begin{array}{l}\text { Infecciones Rickettsiales por garrapatas y pulgas en cuatro poblaciones fronterizas de Perú; } \\
\text { Loreto, Madre de Dios, Tumbes y Tacna. }\end{array}$ & & $X$ & $X$ \\
\hline 8 & $\begin{array}{l}\text { Perfil epidemiológico de la población aledaña al proyecto minero Las Bambas en relación a } \\
\text { enfermedades transmisibles y por exposición a contaminantes ambientales. Apurímac } 2006 .\end{array}$ & & $X$ & $X$ \\
\hline
\end{tabular}


el formato de presentación establecido en las bases del fondo concursable.

Al final de la fase presencial se formularon 28 protocolos de investigación, siete protocolos de la UN de Cajamarca, diez de la UN Jorge Basadre Grohmann de Tacna y once de la UN San Antonio Abad de Cusco. Las líneas temáticas cubiertas por dichos protocolos fueron nutrición, enfermedades trasmisibles, enfermedades no trasmisibles, salud ambiental y ocupacional, mortalidad materna y dos protocolos de ciencias básicas-aplicadas.

\section{HACIA UN SISTEMA NACIONAL DE INVESTIGACIÓN EN SALUD}

Un sistema de investigación en salud es definido como las personas, instituciones y procesos cuyo propósito primario en relación con la investigación es generar conocimiento de alta calidad que pueda ser usado en promover, restaurar y mantener el estado de salud de la población ${ }^{(20)}$. Si bien el Perú cuenta con un sistema nacional de investigación en salud aún incipiente; hay instituciones peruanas con el potencial para conformar un sistema avanzado y fortalecido (21); estas se agrupan en instituciones del Estado, universidades públicas y privadas, servicios de salud (hospitales, institutos especializados) y organizaciones independientes ${ }^{(19)}$. Si bien estas instituciones podrían conformar un sistema de investigación, estas no se encuentran articuladas ni se reconocen como instituciones de investigación. ${ }^{(15)}$

Las UP están involucradas en el sistema de investigación $y$, por consiguiente, participan activamente en el desarrollo de investigación. Según Boza ${ }^{(4)}$, una de las funciones de las universidades es desarrollar un plan de investigación que permita materializar sinergias y concretar oportunidades con especialistas de otras universidades a favor del desarrollo de las zonas impactadas por la minería. En este sentido, las UP tienen como tarea a largo plazo propiciar el uso responsable del canon en el marco de un sistema nacional de investigación en salud. El INS, mediante la estrategia descrita en este artículo, brinda asistencia técnica a las universidades aproximándolas a las prioridades nacionales y regionales de investigación en salud, y a un enfoque multidisciplinario, transdisciplinario e interinstitucional de la investigación; compartiendo los derechos de autor de protocolos de investigación en salud pública.

Esta experiencia reveló varias limitaciones en su implementación, las cuales podemos clasificar en limitaciones de recursos humanos, de la infraestructura, normativa y actitudinal. Dentro de las limitaciones de recursos humanos podemos mencionar: limitadas competencias en metodología de la investigación, búsqueda bibliográfica, redacción científica y gestión de la investigación. En limitaciones de infraestructura encontramos la falta de acceso a bases de datos y repositorios científicos, falta de laboratorios equipados para el perfil epidemiológico de la región, falta de sistemas informáticos de apoyo a la gestión en investigación. Dentro de las limitaciones de normativa tenemos la debilidad en los reglamentos de fondos concursables, ausencia de la estructura administrativa para el manejo de fondos para investigación, normativa que desincentiva a los docentes para la labor de investigación. Finalmente, en limitaciones actitudinales están la visión personalizada y aislada de investigación que tienen la mayoría de docentes de las UP, falta de trabajo multidisciplinario e interinstitucional, débil articulación para la investigación colaborativa con regiones de problemas sanitarios similares, entre otros. Estas limitaciones no permiten que las UP alberguen investigación científica de calidad. El débil y obsoleto componente administrativo y normativo existente, con respecto al desarrollo de investigación, mengua en gran manera las aisladas iniciativas de investigadoresdocentes universitarios y tal situación, como se observó en la experiencia, dificulta el desarrollo de protocolos y su posterior ejecución en caso se consiga acceder al financiamiento del canon.

Una debilidad de la estrategia fue que solo se enfocó en dos de las cuatro limitaciones mencionadas en el párrafo anterior. No se pudo realizar cambios en los componentes infraestructura y normativo; sin embargo, mediante acuerdos suscritos entre el INS y las UP, se dejaron recomendaciones y sugerencias respecto a este último componente, como actualizar y modificar los reglamentos vigentes a favor de la ejecución de investigación, entablar convenios específicos con instituciones públicas y privadas de la región, y establecer las facilidades necesarias a los investigadores universitarios, entre otras.

\section{CONCLUSIONES}

El fortalecimiento de la investigación científica en las UP es una tarea que debería implementarse a través de una política de desarrollo de investigación científica, la cual debe contemplar cuatro aspectos básicos: la infraestructura (física, administrativa y normativa); los investigadores, las prioridades de investigación y las fuentes de financiamiento, entre ellas las del canon, constituyendo esta forma de financiamiento una gran oportunidad para el desarrollo de investigación.

Esta estrategia marca una experiencia importante de trabajo conjunto entre el INS y las UP, producto de 
ella se pusieron en evidencias debilidades y carencias específicas que dificultan el establecimiento de un sistema nacional de investigación en salud. Si bien la presente estrategia tiene debilidades y no pretende ser la solución definitiva a la compleja problemática nacional de investigación en salud, bien podría ser replicada -con mejoras- en otras regiones como parte de un plan coherente, ético, integrador, académico-científico y social; en el marco de una política de desarrollo de investigación científica.
Contribuciones de autoría: FRR, CCS, MES estuvieron a cargo de la de sistematización de la estrategia, FRR, MES recolectaron y analizaron la información disponible. Todos los autores participaron en la redacción del borrador del artículo, revisaron críticamente el artículo y aprobaron la versión final.

Fuentes de financiamiento: Instituto Nacional de Salud.

Conflicto de interés: los autores forman parte de la dirección del Instituto Nacional de Salud.

\section{REFERENCIAS BIBLIOGRÁFICAS}

1. Perú, Tribunal Constitucional. Presupuesto Público. Articulo 77. En: Constitución Política del Perú de 1993. Lima: TC; 1993.

2. Corporación Financiera Internacional (IFC). Manual de gestión del canon. Programa Canon. Herramienta para la gestión de las inversiones municipales. Lima: IFC/USAID; 2009.

3. Perú, Congreso de la República. Ley del Canon. Ley 27506. 9 de julio de 2001.

4. Boza B. Canon minero: ¿caja chica o palanca para el desarrollo? Lima: CAD Ciudadanos al Día - Sociedad Nacional de minería, petróleo y energía; 2006.

5. Perú, Congreso de la República. Ley 28322, Numeral 5.2, modificado por el Artículo 2, publicada el 10 de agosto de 2004,.

6. Perú, Congreso de la República. Ley 28077, Numeral modificado por el Artículo 4, publicada el 26 de septiembre de 2003.

7. Perú, Congreso de la República. Ley 29626, Ley del Presupuesto del Sector Público para el Año Fiscal 2011 Art. 17 , numerales 17.1 y 17.2

8. Perú, Congreso de la República. Ley 29788, Ley que modifica la Ley 28258. Ley de Regalía Minera. 28 de septiembre de 2011.

9. Perú, Congreso de la República. Ley 28258, Ley de Regalía Minera. 03 de junio de 2004.

10. Perú, Congreso de la República. Ley 29465, Ley de Presupuesto del Sector Público para el Año Fiscal 2010. 08 de diciembre de 2009.
11. Perú, Congreso de la República. Ley 29812. Ley del Presupuesto del Sector Público para el Año Fiscal 2012. 09 de diciembre de 2012.

12. Sociedad Nacional de Minería, Petróleo y Energía. Reporte del Canon Minero [Internet]. Lima: 2011 [citado el 12 de junio de 2012]. Disponible en: http://www.snmpe.org.pe/informes-ypublicaciones-snmpe/canon/cuadrosestadisticos/217-reporte-canonminero-2011.html

13. Garfias M. La investigación en la universidad pública regional y los fondos del Canon 2004-2008. Informe Final de Investigación. Lima: Institutos de Estudios Peruanos; 2009.

14. UNESCO. Unesco science report 2010. The current Status of Science around the world. Paris: Unesco; 2010.

15. Mahmood S, Hort K, Ahmed S, Salam M, Cravioto A. Strategies for capacity building for health research in Bangladesh: Role of core funding and a common monitoring and evaluation framework. Health Res Policy Syst. 2011;9:31.

16. Red de Indicadores de Ciencia y Tecnologia (RICYT) [Internet]. Buenos Aires: RICYT; c2009 [citado el 12 de junio del 2012]. Disponible en: http://bd.ricyt.org/explorer.php/ query/submit ?country[]=PE\&syear= 1990\& 2 year $=2009$

17. Perú, Presidencia del Consejo de Ministros, Consejo Nacional de Ciencia, Tecnología e Innovación Tecnológica. Informe de la Comisión Consultiva para la Ciencia Tecnología e Innovación CTI [Internet]. Lima: CONCYTEC; 2012 [citado el 12 de junio de 2012]. Disponible en: http:// portal.concytec.gob.pe/index.php/ concytec/comision-consultiva.html

18. Scimago Institutions Rankings (SIR). Ranking Iberoamericano SIR 2012 [Internet]. SIR; c2012. Disponible en: http://www.scimagoir.com/pdf/ ranking_iberoamericano_2012.pdf

19. Instituto Nacional de Salud. Resolución Jefatural 099 - 2012-J-OPE/INS Sector Salud. Lima, Perú. 16 de abril de 2012.

20. Pang T, Sadana R, Hanney S, Bhutta ZA, Hyder AA, Simon J. Knowledge for better health: a conceptual framework and foundation for health research systems. Bull World Health Organ. 2003,81(11):815-20.

21. Conceicao C, McCarthy M. Public health research systems in the European Union. Health Res Policy Syst. 2011;9:38.

22. Conceicao C, Leandro A, McCartht M. National support to public health research: a survey of European ministries. BMC Public Health. 2009,9:203.

23. McCarthy M, Harvey G, Conceição C, la Torre G, Gulis G. Comparing publichealth research priorities in Europe. Health Res Policy Syst. 2009;7:17.

Correspondencia: Franco Romani Romani

Dirección: Calle Cápac Yupanqui 1400.

Lima 11, Perú.

Teléfono: (511) 990630678

Correo electrónico:fromanir@gmail.com 